\title{
Synthesis of the Antioxidant Glutathione in Neurons: Supply by Astrocytes of CysGly as Precursor for Neuronal Glutathione
}

\author{
Ralf Dringen, Brigitte Pfeiffer, and Bernd Hamprecht \\ Physiologisch-chemisches Institut der Universität, D-72076 Tübingen, Germany
}

Deficiency of the antioxidant glutathione in brain appears to be connected with several diseases characterized by neuronal loss. To study neuronal glutathione metabolism and metabolic interactions between neurons and astrocytes in this respect, neuron-rich primary cultures and transient cocultures of neurons and astroglial cells were used. Coincubation of neurons with astroglial cells resulted within $24 \mathrm{hr}$ of incubation in a neuronal glutathione content twice that of neurons incubated in the absence of astroglial cells. In cultured neurons, the availability of cysteine limited the cellular level of glutathione. During a $4 \mathrm{hr}$ incubation in a minimal medium lacking all amino acids except cysteine, the amount of neuronal glutathione was doubled. Besides cysteine, also the dipeptides CysGly and $\gamma$ GluCys were able to serve as glutathione precursors and caused a concentration-dependent increase in glutathione content. Concentrations giving half-maximal effects were 5, 5, and $200 \mu \mathrm{M}$ for cysteine, CysGly, and $\gamma$ GluCys, respectively. In the transient cocultures, the astroglia-mediated increase in neuronal glutathione was suppressed by acivicin, an inhibitor of the astroglial ectoenzyme $\gamma$-glutamyl transpeptidase, which generates CysGly from glutathione. These data suggest the following metabolic interaction in glutathione metabolism of brain cells: the ectoenzyme $\gamma$-glutamyl transpeptidase uses as substrate the glutathione released by astrocytes to generate the dipeptide CysGly that is subsequently used by neurons as precursor for glutathione synthesis.

Key words: astrocytes; CysGly; glutathione; metabolic coupling; neurons; neurodegeneration; oxidative stress
Evidence is growing that glutathione plays an important role in the detoxification of reactive oxygen species in brain. Deficiencies in brain glutathione metabolism appear to be connected with several neurodegenerative diseases (Bains and Shaw, 1997). For example, a lowered glutathione content has been found in the substantia nigra pars compacta of patients who suffered from Parkinson's disease (Sofic et al., 1992; Sian et al., 1994). In several animal models, it has been demonstrated that glutathione is essential for normal brain function. In newborn rats, induction of glutathione deficiency by application of buthionine sulfoximine (BSO), an inhibitor of the first step in glutathione synthesis, caused mitochondrial damage in brain (Jain et al., 1991). Furthermore, reduction of the brain glutathione content by BSO enhanced the toxic effects of insults, which are discussed to act by generation of reactive oxygen species, i.e., reperfusion after ischemia (Mizui et al., 1992) or treatment with 6-hydroxydopamine (Pileblad et al., 1989) and 1-methyl-4-phenylpyridinium (Wüllner et al., 1996).

On the cellular level, neurons are considered to contain less glutathione than astroglial cells (for review, see Cooper, 1997). In cell culture, values for the amount of glutathione in neurons have been reported. However, these values vary in a range from $<1$ $\mathrm{nmol} / \mathrm{mg}$ protein (Raps et al., 1989) to $40 \mathrm{nmol} / \mathrm{mg}$ protein (Pileblad et al., 1991). Recently it has been demonstrated that the amount of glutathione in neurons and astroglial cells varies with the brain region from which the cells have been prepared. Neurons in cultures prepared from the cortex contain less glutathione

\footnotetext{
Received Aug. 10, 1998; revised Oct. 26, 1998; accepted Oct. 27, 1998.

We thank Dr. Heinrich Wiesinger for critically reading this manuscript.

Correspondence should be addressed to Dr. Ralf Dringen, Physiologischchemisches Institut der Universität, Hoppe-Seyler-Strasse 4, D-72076 Tübingen, Germany.

Copyright (C) 1999 Society for Neuroscience $\quad 0270-6474 / 99 / 190562-08 \$ 05.00 / 0$
}

than astroglial cultures from cortex. In contrast, neuronal and astroglial cultures prepared from striatum or mesencephalon contain almost identical levels of glutathione (Langeveld et al., 1996).

So far little is known about the metabolism of glutathione in brain neurons. As in many other cell types (Bannai and Tateishi, 1986), the availability of cysteine appears to limit the glutathione content in cultured neurons. In culture, it has been demonstrated that neurons are unable to take up cystine (Sagara et al., 1993) and, therefore, rely on the presence of cysteine as substrate for glutathione synthesis (Sagara et al., 1993; Kranich et al., 1996). It has been suggested that astrocytes provide the cysteine essential for neuronal glutathione synthesis by intracellular reduction of cystine and subsequent release of cysteine to neurons (Sagara et al., 1993). Indeed, the presence of astroglial cells increased strongly the content of glutathione in neurons (Bolanos et al., 1996), supporting the idea of a metabolic interaction between astrocytes and neurons regarding glutathione metabolism.

The data presented here demonstrate that the dipeptide CysGly is an excellent precursor of neuronal glutathione. The results suggest that astroglial cells generate this precursor via their ectoenzyme $\gamma$-glutamyl transpeptidase from the glutathione that they release.

Part of this work has been published in abstract form (Dringen et al., 1998b).

\section{MATERIALS AND METHODS}

Materials. DMEM and horse serum were obtained from Life Technologies (Eggenstein, Germany). CysGly and $\gamma$ GluCys were obtained from Bachem (Bubendorf, Switzerland). Fetal calf serum, glutathione (GSH), glutathione disulfide (GSSG), glutathione reductase from yeast (EC 1.6.4.2), insulin, and NADH were purchased from Boehringer Mannhein (Mannheim, Germany). NADPH was from Applichem (Darmstadt, Germany). Acivicin, bovine serum albumin, BSO, cytosine arabinoside, 5,5'-dithio-bis(2-nitrobenzoic acid) (DTNB), poly-D-lysine, progesterone, putrescine, transferrin, and 5-sulfosalicylic acid were obtained from 


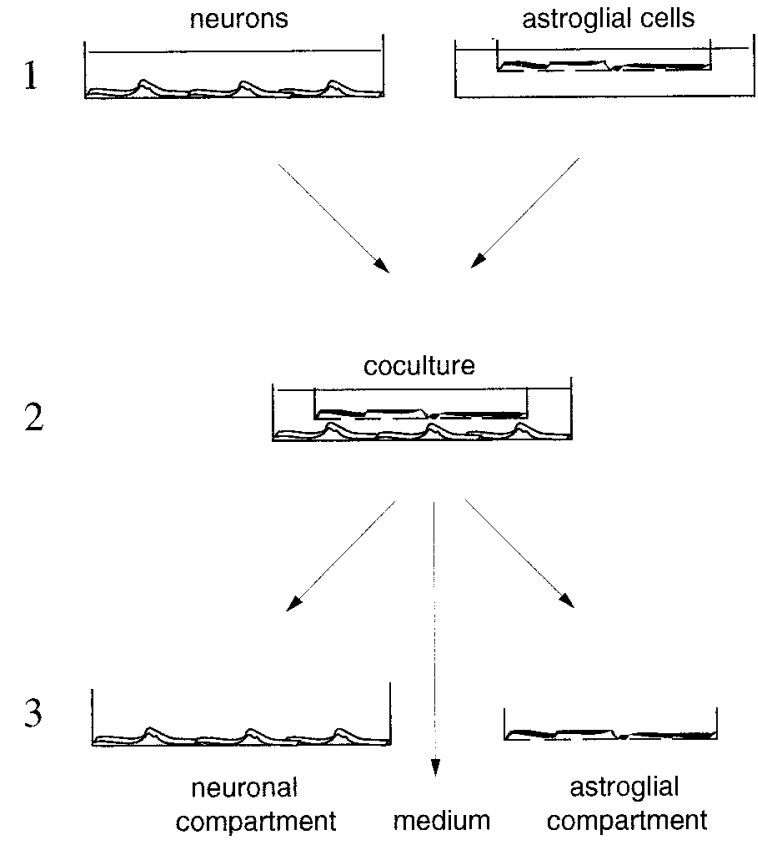

Figure 1. Transient cocultures. 1, Neuron-rich and astroglia-rich primary cultures were prepared and maintained as described in Materials and Methods. The astroglial cells were seeded and cultivated on Anopore membranes, permeable for low molecular weight compounds. 2, After separate incubation of the neuronal and astroglial cultures, the coculture was initiated by transfer of an astroglia-containing culture insert to a well containing cultured neurons. 3, After an appropriate incubation period, glutathione levels were determined in the two compartments of the coculture and in the coculture medium.

Sigma (Deisenhofen, Germany). Sodium pyruvate and sodium selenite were purchased from Fluka (Neu-Ulm, Germany). L-Cysteine, streptomycine sulfate, penicillin G, and Triton X-100 were from Serva (Heidelberg, Germany). All other chemicals, of the highest purity available, were obtained from Merck (Darmstadt, Germany). Cell culture dishes ( $35 \mathrm{~mm}$ in diameter), 6-well dishes, cell culture inserts with Anopore membranes ( $25 \mathrm{~mm}$ in diameter), and 96-well microtiter plates were from Nunc (Wiesbaden, Germany).

Neuron-rich primary cultures. Neuron-rich primary cultures were prepared from the brains of embryonic Wistar rats and maintained as described (Löffler et al., 1986). Briefly, the brains of the embryonic day 16 (E16) rats were prepared and mechanically disrupted by passing through nylon meshes with 135 and $20 \mu \mathrm{m}$ pores. One million viable cells were seeded into poly-D-lysine-coated plastic culture dishes $(35 \mathrm{~mm}$ in diameter) in $2 \mathrm{ml}$ of $90 \%$ DMEM and $10 \%$ horse serum containing penicillin and streptomycin (PS; $20 \mathrm{U} / \mathrm{ml}$ penicillin $\mathrm{G}$ and $20 \mu \mathrm{g} / \mathrm{ml}$ streptomycin sulfate). After $24 \mathrm{hr}$, the cells were washed with $2 \mathrm{ml}$ of DMEM/PS and then incubated in $2 \mathrm{ml}$ of glia-conditioned DMEM/PS (Löffler et al., 1986) containing the five components particular to medium N2 (Bottenstein and Sato, 1979). After 3 d in culture, the cells were treated for $24 \mathrm{hr}$ with cytosine arabinoside in a final concentration of 5 $\mu \mathrm{M}$. Subsequently, the cells were incubated in glia-conditioned DMEM/ $\mathrm{PS} / \mathrm{N} 2$. The cultures were used at an age of 5-7 d. In this range, the specific glutathione content did not depend on the age of the culture. The neuron-rich primary cultures contain minor contaminations of astroglial cells but no oligodendroglial or ependymal cells (Löffler et al., 1986).

Transient cocultures. Astroglia-rich primary cultures were prepared as described (Hamprecht and Löffler, 1985) by seeding 1 million viable cells in $25 \mathrm{~mm}$ cell culture inserts (Nunc) on an Anopore membrane with a pore size of $20 \mathrm{~nm}$ and incubating in $4 \mathrm{ml}$ 90\% DMEM and $10 \%$ fetal calf serum/PS. The culture medium was renewed every $7 \mathrm{~d}$. At a culture age between 17 and $21 \mathrm{~d}$, the transient coculture was initiated by transferring a cell culture insert with astroglial cells into a well of a 6-well plate containing a neuron-rich primary culture of a culture age between 5 and $7 \mathrm{~d}$ (Fig. 1). These neuron-rich cultures were prepared as described above. We seeded 1.5 million viable cells per well of a poly-D-lysinecoated 6-well dish. The membrane of the insert allows traffic of small, diffusible substances between the cells of the two culture types but prevents cell contacts. The protein content of the neuronal and astroglial compartment at the onset of the coculture was $143 \pm 34 \mu \mathrm{g}$ and $264 \pm 48$ $\mu \mathrm{g}$ of protein per well or cell culture insert, respectively $(n=15$, from five cocultures).

Experimental incubation. The culture medium of neuron-rich primary cultures (prepared on plastic dishes $35 \mathrm{~mm}$ in diameter) was replaced by $2 \mathrm{ml}$ of a minimal medium (MM+) containing (in mM) $44 \mathrm{NaHCO}_{3}, 110$ $\mathrm{NaCl}, 1.8 \mathrm{CaCl}_{2}, 5.4 \mathrm{KCl}, 0.8 \mathrm{MgSO}_{4}, 0.92 \mathrm{NaH}_{2} \mathrm{PO}_{4}$, and 5 glucose adjusted with $\mathrm{CO}_{2}$ to $\mathrm{pH} 7.4$ ), containing the amino acids and dipeptides indicated. The cells were incubated for up to $6 \mathrm{hr}$ in the incubator (Heraeus, Hanau, Germany) containing a humidified atmosphere of $10 \%$ $\mathrm{CO}_{2}$ and $90 \%$ air. The incubation was terminated by washing the cells twice with $2 \mathrm{ml}$ of ice-cold PBS (10 mM potassium phosphate buffer, $\mathrm{pH}$ 7.4, containing $150 \mathrm{~mm} \mathrm{NaCl}$ ) to completely remove sulfhydryl-groupcontaining compounds that might interfere with the glutathione assay. Subsequently, the cells were lysed in $200 \mu \mathrm{l}$ of $1 \%(\mathrm{w} / \mathrm{v})$ sulfosalicylic acid on ice for $2 \mathrm{~min}$. The cell lysates were scraped off the dish, and, after centrifugation $(1 \mathrm{~min}, 16000 \times g)$, the supernatant was used for measuring the content of total glutathione (GSx) or GSSG.

The cells of the transient coculture were incubated in a total volume of $2 \mathrm{ml} \mathrm{DMEM} / \mathrm{PS}$ in the presence or the absence of acivicin $(100 \mu \mathrm{M})$. All incubations were performed in the cell incubator containing a humidified atmosphere of $10 \% \mathrm{CO}_{2}$ and $90 \%$ air. The coculture was terminated by collecting the coculture medium, washing the cells of both the astroglial and the neuronal compartment twice with $2 \mathrm{ml}$ of ice-cold PBS, and lysing the cells with $200 \mu \mathrm{l}$ of $1 \%$ (w/v) sulfosalicylic acid. The lysates were collected as described above.

Glutathione assay. GSx (amount of GSH plus 2 times amount of GSSG) was measured in a microtiter plate assay, as described previously (Dringen and Hamprecht, 1996). The standards were carried through exactly the same procedure as the cell extracts. Ten microliters of the cell lysates or GSSG standards $(0-500$ pmol of GSx/10 $\mu$ l) were transferred into 90 $\mu \mathrm{l}$ of water placed in wells of microtiter plates. After addition of $100 \mu \mathrm{l}$ reaction mixture $(0.1 \mathrm{M}$ sodium phosphate buffer, $\mathrm{pH} 7.5$, containing 1 mM EDTA, $0.3 \mathrm{~mm}$ DTNB, $0.4 \mathrm{~mm}$ NADPH, and $1 \mathrm{U} / \mathrm{ml}$ glutathione reductase), the increase in absorbance at $405 \mathrm{~nm}$ was detected in $15 \mathrm{sec}$ intervals over a range of $2.5 \mathrm{~min}$ using a microtiter plate reader (Titertek Plus MS212; ISN Biomedicals, Meckenheim, Germany). Glutathione contents were evaluated with the aid of the software delivered with the plate reader by using a calibration curve established with standard samples. Because the cells were washed twice with PBS before the cells were lysed, the remaining sulfhydryl compounds were present in an amount too low to interfere with the detection of glutathione in the lysates.

GSSG was quantified after derivatization of reduced glutathione with 2-vinylpyridine (2VP; Griffith, 1980), as described previously (Dringen and Hamprecht, 1996). Briefly, $130 \mu \mathrm{l}$ of the protein-free supernatant was mixed with $5 \mu \mathrm{l} 2 \mathrm{VP}$ and adjusted with $0.2 \mathrm{M}$ Tris to a $\mathrm{pH}$ value between 5 and 7. Standard amounts of GSSG were treated the same way. After 1 $\mathrm{hr}$ incubation at room temperature, $10 \mu \mathrm{l}$ of the $2 \mathrm{VP}$-treated samples or standards were assayed as described above using a calibration curve established between 0 and 50 pmol of GSSG per well. The extinction at $405 \mathrm{~nm}$ was followed over $5 \mathrm{~min}$ in $30 \mathrm{sec}$ intervals.

Determination of cell viability. Cell viability was assessed by determining the activity of lactate dehydrogenase $(\mathrm{LDH})$ in the incubation medium, as previously described (Dringen et al., 1998a). Fifty microliters of the incubation medium collected from the dishes after the incubation of the cells were added to $150 \mu \mathrm{l}$ of $80 \mathrm{~mm}$ Tris/ $\mathrm{HCl}$ buffer, $\mathrm{pH} 7.2$, containing $200 \mathrm{~mm} \mathrm{NaCl}$ in a well of a microtiter plate. After addition of $200 \mu \mathrm{l}$ reaction mixture $(80 \mathrm{~mm}$ Tris/ $\mathrm{HCl}$ buffer, $\mathrm{pH} 7.2$, containing 200 $\mathrm{mm} \mathrm{NaCl}, 3.2 \mathrm{~mm}$ pyruvate, and $0.4 \mathrm{~mm} \mathrm{NADH})$, the decrease in the absorbance at $340 \mathrm{~nm}$ was recorded for up to $10 \mathrm{~min}$ in $30 \mathrm{sec}$ intervals at room temperature using the microtiter plate reader. The LDH activity in the incubation buffer was compared with the LDH activity in incubation medium after total lysis of the cells by an incubation at room temperature (30 $\mathrm{min})$ after the addition of Triton X-100 to $1 \%(\mathrm{w} / \mathrm{v})$ final concentration. Zero percent viability corresponds to $100 \%$ LDH activity in the buffer.

Determination of protein content. The content of glutathione per dish of a neuron-rich or astroglia-rich primary culture was normalized to the protein content of this dish. For this purpose, the precipitates of the sulfosalicylic acid precipitation were dried and resuspended in $0.5 \mathrm{M}$ $\mathrm{NaOH}$. An aliquot part of the solution was analyzed for its protein 


\begin{tabular}{|c|c|c|c|c|}
\hline $\begin{array}{l}\text { Incubation time } \\
\text { (hr) }\end{array}$ & Presence of & $\begin{array}{l}\text { GSx content } \\
(\mathrm{nmol} \mathrm{GSx} / \mathrm{mg} \text { protein })\end{array}$ & $\begin{array}{l}\text { GSSG content } \\
\text { (nmol GSx/mg protein) }\end{array}$ & Viability (\%) \\
\hline 0 & & $15.9 \pm 0.3$ & $0.4 \pm 0.3$ & $100.0 \pm 8.6$ \\
\hline 4 & Gln & $13.6 \pm 0.5$ & $0.2 \pm 0.1$ & $101.0 \pm 1.7$ \\
\hline 4 & Gln + Cys + Gly & $38.4 \pm 1.0$ & $0.1 \pm 0.2$ & $94.4 \pm 1.7$ \\
\hline 4 & Gln + CysGly & $39.2 \pm 0.9$ & $0.1 \pm 0.2$ & $96.7 \pm 0.5$ \\
\hline 4 & $\gamma$ GluCys + Gly & $37.9 \pm 0.6$ & $0.4 \pm 0.2$ & $94.0 \pm 4.0$ \\
\hline
\end{tabular}

Neuron-rich primary cultures (5-d-old) were incubated for $4 \mathrm{hr}$ in $\mathrm{MM}+$ containing the amino acids or dipeptides indicated in concentrations of $1 \mathrm{~mm}$. The data presented are triplicates \pm SD. The experiment was repeated on an independent culture with comparable results.

content according to the method described by Lowry et al. (1951). Bovine serum albumin was used as a standard.

Statistical analysis. Because the absolute values for GSx content of the neuron cultures as well as the degree of increase of glutathione content after application of cysteine or a cysteine substitute varied strongly between independently prepared cultures, an inclusion of data obtained from different cultures for one statistical analysis was not always appropriate. However, within one culture the GSx values obtained from replica plates were almost identical $(\mathrm{SD}<7 \%)$. Therefore, unless stated otherwise, the data presented on the glutathione content of neuron-rich primary cultures represent triplicates \pm SD obtained from replica plates of one culture. All results shown were reproduced with comparable results on at least one independently prepared culture.

\section{RESULTS}

\section{Glutathione content in neuron cultures}

The total glutathione content (GSx) of neuron-rich primary cultures varied among independently prepared cultures. In 27 individual experiments performed in triplicates of replica plates on 15 independently prepared cultures, GSx values were obtained between the extreme values $13.7 \pm 0.3$ and $44.2 \pm 0.9 \mathrm{nmol}$ of $\mathrm{GSx} / \mathrm{mg}$ protein with an average amount of $23.7 \pm 6.0 \mathrm{nmol}$ of $\mathrm{GSx} / \mathrm{mg}$ protein. However, the glutathione content in replica plates of one culture was almost constant with an average SD $<7 \%$ (27 experiments). The amount of GSx found represented almost exclusively GSH. GSSG was encountered only in small amounts in the range of the detection limit of the method used for its determination. The GSSG content accounted for maximal $2.5 \%$ of the total glutathione content (Table 1).

\section{Glutathione content in transient neuron-astroglia cocultures}

To study metabolic interactions between brain neurons and astrocytes in the synthesis of the antioxidant glutathione, a transient coculture system was established (Fig. 1). After the incubation periods indicated, the neuronal and astroglial compartments were separated from each other (Fig. 1), and glutathione content was determined in the cells of both compartments as well as in the incubation medium. After onset of the incubation, the glutathione content of neurons incubated in the absence of astroglial cells remained almost constant (Fig. $2 A$ ). In contrast, after application of astroglial cells, the glutathione content in the neuronal compartment increased (Fig. $2 A$ ) and reached, after $24 \mathrm{hr}$ of incubation, twice the initial level of glutathione or $165 \%$ of the glutathione amount of cells incubated in the absence of astroglial cells (Figs. $2 A, 7$ ). In contrast to the glutathione content in the neuronal compartment of the coculture, that in the astroglial compartment remained almost unchanged during the coculture with neurons (Fig. $2 B$ ). In the medium of the coculture, glutathione was hardly detectable during the first $10 \mathrm{hr}$ of the incubation.
However, after $24 \mathrm{hr}$ of incubation, the glutathione concentration in the medium exceeded $2 \mu \mathrm{M}$ (Fig. 2C, Table 2).

\section{Glutathione content in neurons after application of glutamine, cysteine, and glycine}

To identify glutathione precursors, the availability of which limit the synthesis of glutathione in neurons, neuron-rich primary cultures were incubated for $4 \mathrm{hr}$ in a minimal medium containing glucose (5 mM) (MM+) and the amino acids indicated (Fig. 3). The high viability of the cells was not altered by this incubation (data not shown). In the absence of amino acids or in the presence of glutamine, glycine, or glutamine plus glycine, no increase in neuronal glutathione content was found in respect to the amount in untreated cells (Fig. 3). In contrast, application of cysteine in the absence or the presence of glycine and/or glutamine caused a significant increase in the amount of neuronal glutathione, at least doubling the content in comparison to that of untreated cells (Fig. 3).

\section{Dipeptides as precursors for neuronal glutathione synthesis}

To study the ability of neurons to use the cysteine-containing dipeptides CysGly and $\gamma \mathrm{GluCys}$ as precursors for glutathione synthesis, the glutathione content of neurons was measured $4 \mathrm{hr}$ after incubation of the cells with the dipeptides. Both dipeptides were able to substitute for cysteine in doubling glutathione content in neurons within $4 \mathrm{hr}$ (Fig. 4, Table 1). After incubation of the cells with cysteine or one of the two dipeptides, the increased glutathione content represented almost exclusively GSH; only minor amounts of GSSG were detectable (Table 1). Under these conditions the viability of the cells was practically unchanged (Table 1).

The first enzyme in glutathione synthesis is the $\gamma$-glutamylcysteine synthetase. To investigate its involvement in the utilization of the dipeptides as precursors for glutathione synthesis in neurons, BSO, an inhibitor of this enzyme (Griffith and Meister, 1979), was applied to neurons together with the dipeptides. The presence of BSO not only completely prevented the increase in neuronal GSx content during incubation with cysteine or one of the dipeptides, but also caused, within $4 \mathrm{hr}$ of incubation compared with the initial content, a decrease of at least $30 \%$ in the neuronal content of glutathione (Fig. 4). This finding for neuronal glutathione synthesis from $\gamma$ GluCys as precursor contrasts with the situation described for kidney (Anderson and Meister, 1983), brain (Pileblad and Magnusson, 1992), and for cultured astroglial cells (Dringen et al., 1997b), in which $\gamma$ GluCys can bypass the $\gamma$-glutamylcysteine synthetase reaction.

The glutathione content of neurons in culture was rapidly 


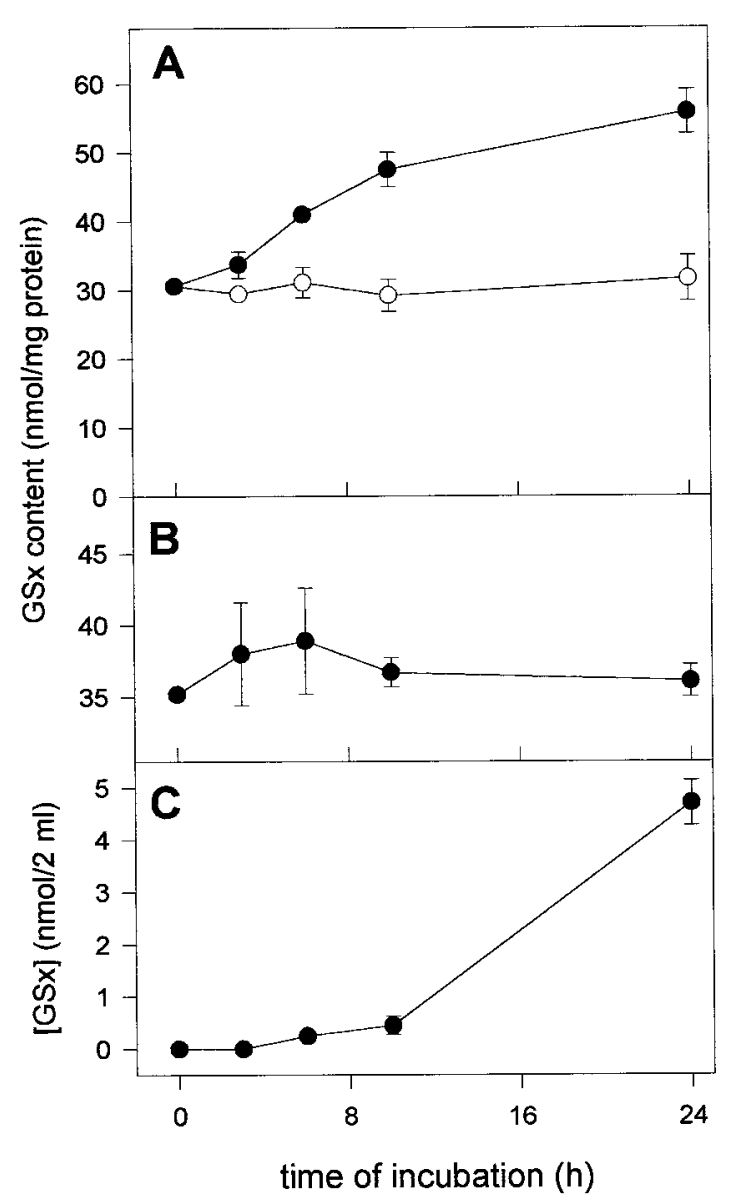

Figure 2. Glutathione content in astroglia-neuron coculture. The neurons were incubated in DMEM/PS in the absence (open circles) or the presence (closed circles) of cell culture inserts containing astroglial cells. The glutathione content was determined at the time points indicated in the neuronal (A, closed circles) and astroglial $(B)$ compartment of the coculture, in neuron-rich cultures incubated in the absence of astroglial cells ( $A$, open circles), and in the incubation medium of the coculture $(C)$. At the onset of the incubation, the primary cultures of neurons and astroglial cells were 6- and 21-d-old, respectively. The data presented are triplicates \pm SD obtained in one experiment. Using independent cultures, the experiment was repeated twice with comparable results.

enhanced after application of dipeptides (Fig. 5). After $1 \mathrm{hr}$ of incubation, neurons incubated with $\gamma$ GluCys contained glutathione levels higher than those found in neurons fed with cysteine or CysGly. Maximal glutathione contents were observed after $\sim 4 \mathrm{hr}$ of incubation if cysteine, CysGly, or $\gamma$ GluCys were fed in concentrations of $1 \mathrm{~mm}$ (Fig. 5). By making the concentrations of cysteine plus glycine, CysGly, or $\gamma$ GluCys limiting for glutathione synthesis, the dependency of the cellular glutathione content on the concentrations of these substrates was determined. Exogenous cysteine plus glycine, or CysGly or $\gamma$ GluCys were used for neuronal glutathione synthesis with half-maximal effects at respective concentrations of about 5, 5, and $200 \mu \mathrm{M}$ (Fig. 6). Maximal glutathione levels were attained at concentrations of $\sim 30 \mu \mathrm{M}$ cysteine plus glycine or CysGly, whereas $\gamma$ GluCys had to be present in a concentration of $1 \mathrm{~mm}$ to raise neuronal glutathione to a maximal level (Fig. 6). In contrast to the presence of dipeptides, cysteine in concentrations higher than $100 \mu \mathrm{M}$ led to a submaximal glutathione content in neurons (Fig. 6). This reduction in maximal glutathione content of neurons incubated with cysteine at a concentration of $1 \mathrm{~mm}(78.2 \pm 8.7 \%)$ rather than with $100 \mu \mathrm{M}(100 \%)$ was reproducible and significant $(p<0.001$; $n=12$ from four experiments) but was not accompanied by a decrease in the cell viability (data not shown).

\section{Effect of acivicin on the GSx content in cocultures}

Because CysGly is the product of a reaction catalyzed by $\gamma \mathrm{GT}$, it is hypothesized that this dipeptide is generated from the GSH released by astroglial cells. Thus, CysGly would be produced in equimolar concentration to the GSH used as substrate, and, subsequently, could become substrate for neuronal glutathione synthesis (see Fig. 8). To test for an involvement of $\gamma \mathrm{GT}$ in the astroglia-induced increase in the neuronal glutathione level in the cocultures, acivicin was applied, an inhibitor of the ectoenzyme $\gamma$ GT (Stole et al., 1994; Dringen et al., 1997a). Presence of acivicin in a concentration of $100 \mu \mathrm{M}$ during an incubation for 24 hr did not significantly alter the glutathione content in neurons compared with cells incubated in the absence of the inhibitor (Fig. 7). However, acivicin completely prevented the astrogliainduced increase in the glutathione content of the neuronal compartment of the coculture (Fig. 7). No statistically significant difference $(p>0.1)$ was observed between the glutathione content of neurons cultivated in the absence of astroglial cells and neurons cultivated in the presence of astroglial cells plus acivicin (Fig. 7). In addition, during incubation of the cocultures with acivicin, the amount of glutathione in the medium after $24 \mathrm{hr}$ of incubation was $234 \pm 67 \%(n=12 ; p<0.001)$ of that found in the medium of cocultures incubated in the absence of acivicin. In all four experiments performed, acivicin raised the level of glutathione in the coculture medium more than it lowered that level in the cocultured neurons (Table 2).

\section{DISCUSSION}

The glutathione content of neuronal cultures reported in the literature varies strongly from one report to another (see intro-

Table 2. Glutathione content of the neuronal compartment and the incubation medium of transient cocultures in the presence $(100 \mu \mathrm{M})$ or the absence $(0 \mu \mathrm{M})$ of acivcin

\begin{tabular}{|c|c|c|c|c|c|c|}
\hline \multirow[b]{2}{*}{ Experiment } & \multicolumn{2}{|c|}{ GSx in media $(\mathrm{nmol} / 2 \mathrm{ml})$} & \multirow[b]{2}{*}{$\Delta$} & \multicolumn{2}{|c|}{ GSx in neurons (nmol/well) } & \multirow[b]{2}{*}{$\Delta$} \\
\hline & + Acivicin & - Acivicin & & + Acivicin & - Acivicin & \\
\hline 1 & $13.6 \pm 5.1$ & $4.6 \pm 1.4$ & 9.0 & $3.1 \pm 0.1$ & $5.9 \pm 0.3$ & -2.7 \\
\hline 2 & $8.5 \pm 0.1$ & $4.4 \pm 0.8$ & 4.1 & $5.6 \pm 0.6$ & $8.7 \pm 0.1$ & -3.1 \\
\hline 3 & $14.6 \pm 0.6$ & $5.6 \pm 0.6$ & 9.0 & $4.8 \pm 0.7$ & $9.4 \pm 0.5$ & -4.6 \\
\hline 4 & $8.8 \pm 0.2$ & $4.7 \pm 0.4$ & 4.1 & $3.8 \pm 0.1$ & $7.4 \pm 0.2$ & -3.6 \\
\hline
\end{tabular}

The data were obtained from four independently prepared cocultures incubated for $24 \mathrm{hr}$. The data represent triplicates \pm SD. $\Delta$ represents the difference between the glutathione contents found after incubation in the presence and absence of acivicin. 


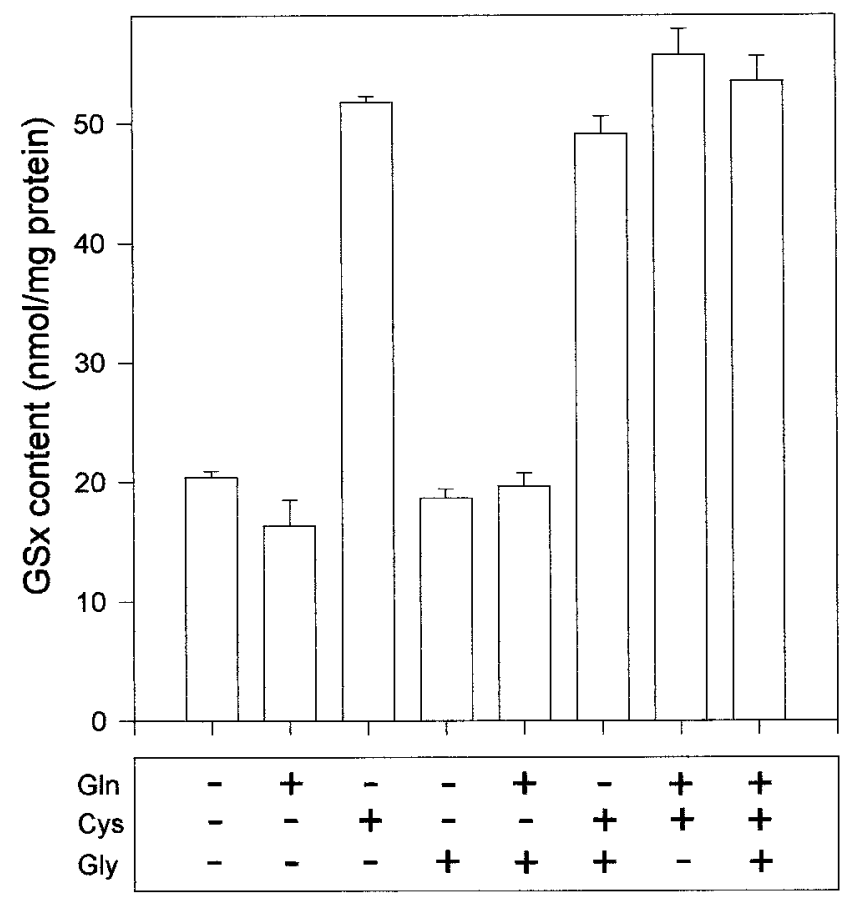

Figure 3. Glutathione content in a 6-d-old neuron-rich primary culture after incubation for $4 \mathrm{hr}$ in $\mathrm{MM}+$ containing the amino acids indicated in concentrations of $1 \mathrm{~mm}$. Before the incubation, the cells contained $23.3 \pm$ $3.3 \mathrm{nmol}$ of $\mathrm{GSx} / \mathrm{mg}$ of protein. The data presented are triplicates $\pm \mathrm{SD}$. The experiment was repeated on an independent culture with comparable results.

ductory remarks). These differences might be attributed to differences in the preparation techniques, to species differences, to the use of different brain areas for the preparation of the cultures (Langeveld et al., 1996), or to different culture conditions. Variations in the composition of the glia-conditioned medium (Löffler et al., 1986) that was used in the present study for the maintenance of the neuron-rich cultures might explain the alterations in the glutathione content of different batches of neurons. The cysteine precursor containing medium conditioned by glial cells was collected from several astroglial cultures and was stored for different time periods before application to the neuron cultures. Therefore, the concentration of unstable glia-derived compounds might differ from batch to batch of the medium. That could be the case for compounds containing sulfhydryl groups and for growth factors, which have been reported to influence neuronal glutathione metabolism (Spina et al., 1992; Pan and Perez-Polo, 1993; Cheng and Mattson, 1995; Mattson et al., 1995).

A glutathione content well above the highest value reported for untreated neurons in culture (40 nmol of $\mathrm{GSx} / \mathrm{mg}$ of protein; Pileblad et al., 1991) was reached, if cysteine, CysGly, or $\gamma$ GluCys was administered to cultured neurons. Because only marginal amounts of GSSG were found in the cultured neurons, these cells appeared not to suffer from oxidative stress during cultivation. This finding is in accord with previous data showing that GSSG accounts for $<1 \%$ of the glutathione in whole brain (Cooper et al., 1980; Rehncrona et al., 1980).

In contrast to cysteine, the availability of glutamine or glycine did not limit neuronal glutathione synthesis. Even in a minimal medium, only cysteine or a cysteine precursor had to be present to double neuronal glutathione levels during $4 \mathrm{hr}$ of incubation, indicating that glutamate/glutamine and glycine were present in

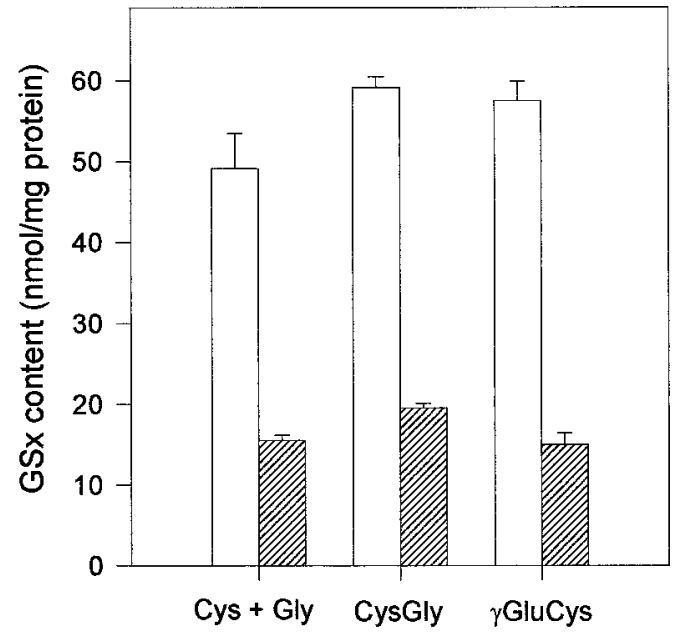

Figure 4. Effect of BSO on the utilization of CysGly and $\gamma$ GluCys for glutathione synthesis in 6-d-old neuron-rich primary cultures. The cells were incubated for $4 \mathrm{hr}$ in MM+ containing Gln + Cys + Gly, Gln + CysGly, or Gly $+\gamma$ GluCys in concentrations of $1 \mathrm{~mm}$ each in the absence (open bars) or the presence (hatched bars) $(0.5 \mathrm{~mm})$ of BSO. Before the incubation, the cells contained $28.0 \pm 0.7 \mathrm{nmol}$ of $\mathrm{GSx} / \mathrm{mg}$ of protein. The data presented are triplicates \pm SD. The experiment was repeated with comparable results using an independent culture.

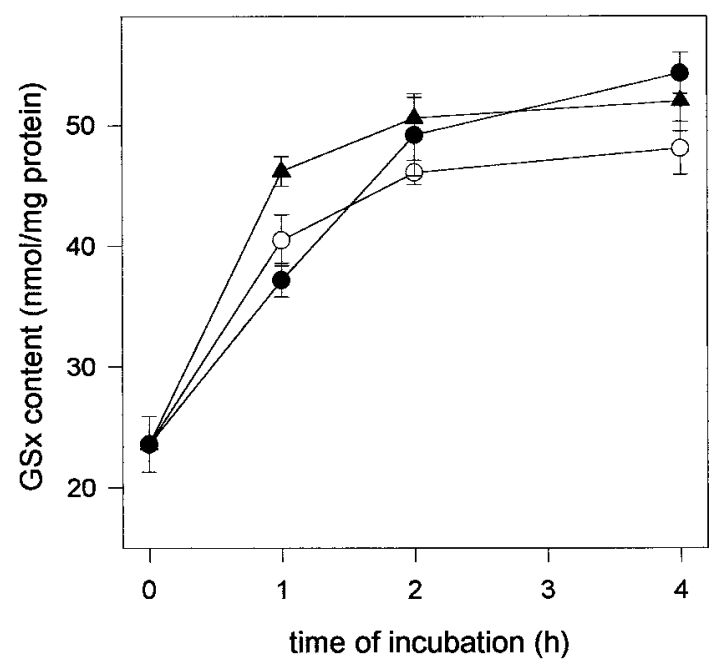

Figure 5. Time course of the glutathione content in 5-d-old neuron-rich primary cultures during incubation with amino acids and/or dipeptides. The cells were incubated for the time periods indicated in MM+ containing glutamine and either cysteine plus glycine (open circles) or CysGly (closed circles), or glycine plus $\gamma$ GluCys (closed triangles). The amino acids or dipeptides were applied in concentrations of $1 \mathrm{~mm}$. The data presented are triplicates \pm SD. Using an independent culture, the experiment was repeated with comparable results.

the cells in concentrations sufficient for glutathione synthesis. Besides cysteine, also the dipeptides CysGly and $\gamma$ GluCys served as precursors for neuronal glutathione. Like cysteine, CysGly was efficiently used by neurons as glutathione precursor in micromolar concentrations. In contrast, $\gamma$ GluCys had to be applied in at least one order of magnitude higher concentration to obtain maximal glutathione levels. The concentrations of cysteine, CysGly, and $\gamma$ GluCys providing half-maximal effects on glutathione synthesis are lower in neurons than in astroglial cells (Dringen et al., 


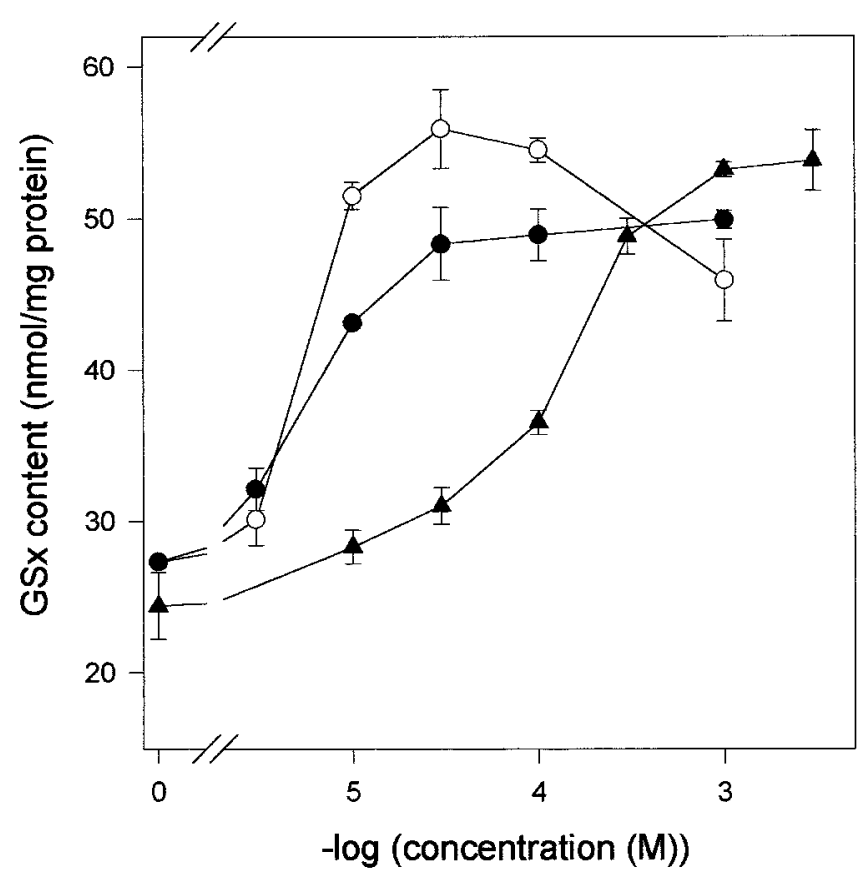

Figure 6. Glutathione content in a 6-d-old neuron-rich primary culture after incubation with amino acids and/or dipeptides. The cells were incubated for $4 \mathrm{hr}$ in $\mathrm{MM}+$ containing glutamine $(1 \mathrm{mM})$ and either cysteine plus glycine (open circles) or CysGly (closed circles) in the concentrations indicated or in $\mathrm{MM}+$ containing glycine $(1 \mathrm{mM})$ plus $\gamma$ GluCys (closed triangles) of varied concentration. Before the incubation the cellular glutathione content was $34.0 \pm 1.5 \mathrm{nmol}$ of $\mathrm{GSx} / \mathrm{mg}$ of protein. The data represent triplicates $\pm \mathrm{SD}$. The experiment was repeated with comparable results using an independently prepared culture.

1997b), indicating that neurons are more efficient in utilizing these compounds as glutathione precursors than astroglial cells.

The inhibition by the $\gamma$-glutamylcysteine synthetase inhibitor BSO of the utilization by neurons of CysGly and $\gamma$ GluCys for glutathione synthesis indicates that both dipeptides must be hydrolyzed before their constituent amino acids serve as substrates for glutathione synthesis. For $\gamma \mathrm{GluCys}$, an extracellular cleavage has to be strongly considered, unless one wants to invoke a futile cycle of intracellular synthesis and degradation of $\gamma$ GluCys in neurons. The decrease in glutathione levels of BSO-exposed neurons in spite of the presence of sufficient glutathione precursors demonstrates a rapid turnover of neuronal glutathione.

The level of cysteine is low in extracellular fluids, because this amino acid is rapidly oxidized by oxygen (Ishii and Bannai, 1985). Therefore, the question arises as to how neurons, cells known to be unable to use cystine, acquire the cysteine essential for glutathione synthesis. Astrocytes could provide a cysteine precursor for neuronal glutathione synthesis. At least in culture, the presence of astroglial cells has been reported to maintain (Sagara et al., 1993) or even increase glutathione levels in cultured neurons (Bolanos et al., 1996). These findings were confirmed in the present study. The glutathione level in the neuronal compartment was doubled within $24 \mathrm{hr}$ in the presence of astroglial cells, indicating that (1) in the absence of astroglial cells at least one precursor for glutathione is limiting neuronal glutathione synthesis, and (2) that a metabolite was provided from the astroglial cells to the neurons able to bypass this limitation. Cysteine has been reported to be released from astroglial cells in cystinecontaining culture medium (Sagara et al., 1993). However, this release has to take place against the sodium gradient, which

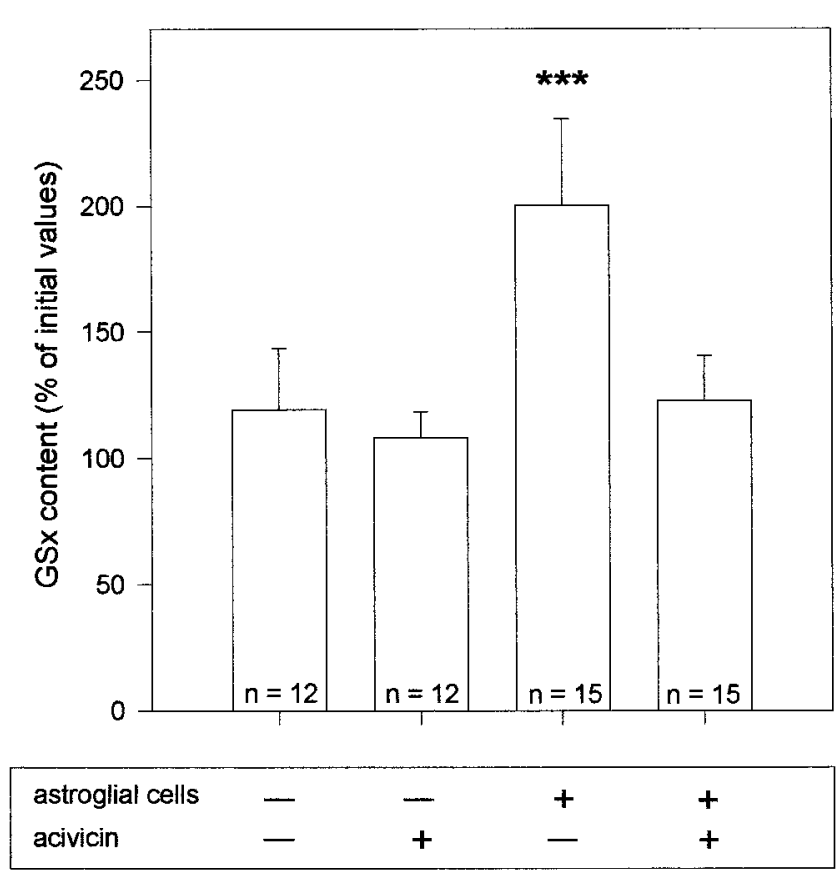

Figure 7. Effect of acivicin and astroglial cells on the glutathione content in neuron-rich primary cultures. Neurons were incubated for $24 \mathrm{hr}$ in DMEM/PS in the presence or the absence of acivicin $(100 \mu \mathrm{M})$ and/or a cell culture insert containing an astroglia-rich primary culture. The GSx values obtained in four or five experiments, each performed in triplicates on independent cultures, were compared with the GSx content of neurons before onset of the incubation. Compared to the glutathione content of untreated neurons and neurons incubated without astroglial cells, with acivicin, or with astroglial cells plus acivicin, the GSx content of neurons after incubation with astroglial cells in the absence of acivicin is highly significantly different $(p<0.00001)$ as analyzed by Student's $t$ test.

enables astroglial cells to efficiently take up cysteine (Sagara et al., 1993). The appearance of cysteine in the culture medium of astroglial cells can also be explained as a consequence of the release of glutathione from astroglial cells (Yudkoff et al., 1990; Sagara et al., 1996; Dringen et al., 1997b), because GSH liberates cysteine from cystine by forming mixed disulfides (Deneke et al., 1995). Therefore, it can be doubted that the cysteine found in the culture medium of astroglial cells was indeed released from astroglial cells.

Recently it has been reported that the GSH released from astroglial cells is partially used as substrate of the astroglial ectoenzyme $\gamma \mathrm{GT}$ (Dringen et al., 1997a). One product of the $\gamma \mathrm{GT}$ reaction is the dipeptide CysGly (Meister et al., 1981; Huseby, 1988), which is used by neurons in micromolar concentrations as precursor for glutathione. This finding suggests the involvement of astroglial $\gamma \mathrm{GT}$ in the generation of the cysteine precursor provided by astroglial cells for neuronal glutathione synthesis (Fig. 8). To prove this hypothesis, the $\gamma \mathrm{GT}$ inhibitor acivicin was applied to the incubation medium of neuron-astroglia cocultures. The presence of acivicin totally prevented the astroglia-induced effect on the glutathione content in the neuronal compartment of the coculture. Although the specificity of acivicin for inhibiting $\gamma$ GT has not been fully established, this finding indicates that $\gamma \mathrm{GT}$ is involved in the supply of a glutathione precursor from astroglial cells to neurons. The finding that neurons incubated for $24 \mathrm{hr}$ with acivicin contain a glutathione level identical to neurons cultured in the absence of acivicin demonstrates that acivicin, in contrast to BSO, did not alter glutathione turnover in neurons by 


\section{Astrocyte}

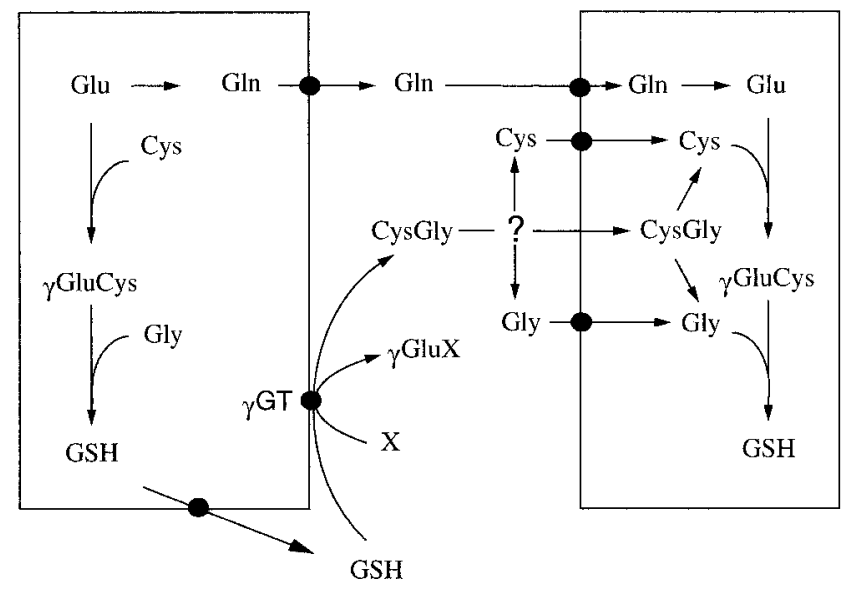

Figure 8. Scheme of the proposed metabolic interaction between neurons and astrocytes regarding glutathione synthesis. The GSH released from astrocytes is substrate for the astroglial ectoenzyme $\gamma$ GT. CysGly, one product of the $\gamma \mathrm{GT}$ reaction, serves as a precursor of neuronal glutathione. It is not known whether the hydrolysis of CysGly occurs in the extracellular space by a neuronal ectopeptidase or after uptake of the dipeptide in the neurons. In addition, glutamine is released from astrocytes and used by neurons as precursor for glutamate necessary for glutathione synthesis. $X$ represents an acceptor of the $\gamma$-glutamyl moiety transferred from glutathione by $\gamma \mathrm{GT}$, the most likely candidates being glutamine and $\mathrm{H}_{2} \mathrm{O}$.

inhibition of glutathione synthesis but rather inhibited $\gamma \mathrm{GT}$ in the astroglial compartment of the coculture. The amount of glutathione in the media preserved by the inhibition of $\gamma \mathrm{GT}$ is at least as high as the increase in the glutathione content in the neuronal compartment in the absence of acivicin. Therefore, in the coculture the CysGly most likely generated by $\gamma$ GT would allow for the increase of glutathione in the neuronal compartment of the coculture. Steady-state concentrations of CysGly in the coculture medium will be at best in the low micromolar range, which will make it difficult to identify this dipeptide in the coculture medium. Such a task might even be more difficult, because the involvement of an ectopeptidase in the utilization of the dipeptide has to be envisaged as an alternative to the uptake of CysGly into neurons (Fig. 8).

Besides the dipeptide CysGly, the second product of the $\gamma \mathrm{GT}$ reaction is the $\gamma$-glutamyl derivative of the second substrate. Excellent substrates of this enzyme are the amino acids glutamine, methionine, and cystine (Tate and Meister, 1974; Thompson and Meister, 1975). In addition, also $\mathrm{H}_{2} \mathrm{O}$ can serve as an acceptor of the $\gamma$-glutamyl moiety (Binkley and Nakamura, 1948; Meister et al., 1981). Further studies are required to show which products besides CysGly are generated by astroglial $\gamma \mathrm{GT}$ and what the functions and the metabolism of these compounds might be in the nervous system.

Besides the supply of a cysteine precursor, other compounds might also be provided to neurons by astroglial cells during coculture. For the increase in glutathione content in the neuronal compartment of the coculture, a contribution of glial-derived growth factors has to be considered, because an influence of neurotrophic factors on neuronal glutathione metabolism has been reported (see above). However, it is somewhat unlikely that such factors released from the astroglial compartment would accumulate within a few hours in the coculture medium to a concentration sufficient to strongly modulate the expression levels of enzymes involved in glutathione synthesis. Although such an effect cannot be ruled out, the availability of a cysteine precursor predominantly limits neuronal glutathione synthesis. Therefore, the role of growth factors in the astroglia-induced increase in neuronal glutathione might be at best a supporting one.

With the release of glutamine by astroglial cells (Robinson et al., 1998) and the extracellular generation of CysGly from glutathione, astroglial cells provide precursors for all three constituting amino acids of glutathione to neurons. For normal brain function, it might be essential that it is not the substrates for the enzymes involved in glutathione synthesis that are directly delivered to neurons, because glutamate, cysteine, and glycine might harm neurons (Olney et al., 1972; Choi, 1988; Kemp and Leeson, 1993; Puka-Sundvall et al., 1995). In contrast, no toxic effects have been reported for glutamine and CysGly.

In conclusion, the data presented here suggest that CysGly generated by $\gamma$ GT from the glutathione released from astrocytes serves as precursor for neuronal glutathione synthesis (Fig. 8). This metabolic interaction between astrocytes and neurons provides several new targets of interference that might be envisaged for therapeutical purposes. Substances able to increase either glutathione release, activity of $\gamma \mathrm{GT}$, or utilization of CysGly by neurons could elevate glutathione concentrations in brain neurons. As a consequence, those neurons would be protected better against oxidative stress.

\section{REFERENCES}

Anderson ME, Meister A (1983) Transport and direct utilization of $\gamma$-glutamylcyst(e)ine for glutathione synthesis. Proc Natl Acad Sci USA 80:707-711.

Bains JS, Shaw CA (1997) Neurodegenerative disorders in humans: the role for glutathione in oxidative stress-mediated neuronal death. Brain Res Rev 25:335-358.

Bannai S, Tateishi N (1986) Role of membrane transport in metabolism and function of glutathione in mammals. J Membr Biol 89:1-8.

Binkley F, Nakamura K (1948) Metabolism of glutathione. I. Hydrolysis by tissues of the rat. J Biol Chem 173:411-421.

Bolanos JP, Heales SJR, Peuchen S, Barker JE, Land JM, Clark JB (1996) Nitric oxide-mediated mitochondrial damage: a potential neuroprotective role for glutathione. Free Radic Biol Med 21:995-1001.

Bottenstein JE, Sato GH (1979) Growth of a rat neuroblastoma cell line in serum-free supplemented medium. Proc Natl Acad Sci USA 76:514-517.

Cheng B, Mattson MP (1995) PDGFs protect hippocampal neurons against energy deprivation and oxidative injury: evidence for induction of antioxidant pathways. J Neurosci 15:7095-7104.

Choi DA (1988) Glutamate neurotoxicity and diseases of the nervous system. Neuron 1:623-634.

Cooper AJL (1997) Glutathione in the brain: disorders of glutathione metabolism. In: The molecular and genetic basis of neurological disease (Rosenberg RN, Prusiner SB, DiMauro S, Barchi RL, Kunk LM, eds), pp 1195-1230. Boston: Butterworth-Heinemann.

Cooper AJL, Pulsinelli WA, Duffy TE (1980) Glutathione and ascorbate during ischemia and postischemic reperfusion in rat brain. $\mathrm{J} \mathrm{Neu-}$ rochem 35:1242-1245.

Deneke SM, Susanto I, Vogel KA, Williams CE, Lawrence RA (1995) Mechanism of use of extracellular glutathione by lung epithelial cells and pulmonary artery endothelial cells. Am J Respir Cell Mol Biol 12:662-668.

Dringen R, Hamprecht B (1996) Glutathione content as an indicator for the presence of metabolic pathways of amino acids in astroglial cultures. J Neurochem 67:1375-1382.

Dringen R, Kranich O, Hamprecht B (1997a) The $\gamma$-glutamyl transpeptidase inhibitor acivicin preserves glutathione released by astroglial cells in culture. Neurochem Res 6:727-733.

Dringen R, Kranich O, Löschmann PA, Hamprecht B (1997b) Use of dipeptides for the synthesis of glutathione by astroglia-rich primary cultures. J Neurochem 69:868-874.

Dringen R, Kussmaul L, Hamprecht B (1998a) Detoxification of exoge- 
nous hydrogen peroxide and organic hydroperoxides by cultured astroglial cells assessed by microtiter plate assay. Brain Res Protocols 2:223-228.

Dringen R, Kranich O, Pfeiffer B, Hamprecht B (1998b) Glutathione metabolism of brain cells: metabolic interaction between astrocytes and neurons. J Neurochem [Suppl] 71:S6A.

Griffith OW (1980) Determination of glutathione and glutathione disulfide using glutathione reductase and 2-vinylpyridine. Anal Biochem 106:207-212.

Griffith OW, Meister A (1979) Potent and specific inhibition of glutathione synthesis by buthionine sulfoximine (S- $N$-butyl homocysteine sulfoximine). J Biol Chem 254:7558-7560.

Hamprecht B, Löffler F (1985) Primary glial cultures as a model system for studying hormone action. Methods Enzymol 109:341-345.

Huseby NE (1988) $\gamma$-Glutamyltranspeptidase. In: Glutamine and glutamate in mammals (Kvamme E, ed), pp 153-163. Boca Raton: CRC.

Ishii T, Bannai S (1985) The synergistic action of the copper chelator bathrocuproine sulphonate and cysteine in enhancing growth of L1210 cells in vitro. J Cell Physiol 125:151-155.

Jain A, Martenson J, Stole E, Auld PAM, Meister A (1991) Glutathione deficiency leads to mitochondrial damage in brain. Proc Natl Acad Sci USA 88:1913-1917.

Kemp JA, Leeson PD (1993) The glycine site of the NMDA receptorfive years on. Trends Pharmacol Sci 14:20-25.

Kranich O, Hamprecht B, Dringen R (1996) Different preferences in the utilization of amino acids for glutathione synthesis in cultured neurons and astroglial cells derived from rat brain. Neurosci Lett 219:211-214.

Langeveld CH, Schepens E, Jongenelen CAM, Stoof JC, Hjelle OP, Ottersen OP, Drukarch B (1996) Presence of glutathione immunoreactivity in cultured neurons and astrocytes. NeuroReport 7:1833-1836.

Löffler F, Lohmann SM, Walkhoff B, Walter U, Hamprecht B (1986) Immunocytochemical characterization of neuron-rich primary cultures of embryonal rat brain cells established by neuronal and glial markers and by monospecific antisera against cyclic nucleotide-dependent protein kinases and the synaptic vesicle protein synapsin I. Brain Res 363:205-211.

Lowry OH, Rosebrough NJ, Farr AL, Randall RJ (1951) Protein measurement with the folin phenol reagent. J Biol Chem 193:265-275.

Mattson MP, Lovell MA, Furukawa K, Markesbery WR (1995) Neurotrophic factors attenuate glutamate-induced accumulation of peroxides, elevation of intracellular $\mathrm{Ca}^{2+}$ concentration, and neurotoxicity and increase antioxidant enzyme activities in hippocampal neurons. J Neurochem 65:1740-1751.

Meister A, Tate SS, Griffith OW (1981) $\gamma$-Glutamyl transpeptidase. Methods Enzymol 77:237-253.

Mizui T, Kinouchi H, Chan PH (1992) Depletion of brain glutathione by buthionine sulfoximine enhances cerebral ischemic injury in rats. Am J Physiol 262:H313-H317.

Olney JW, Ho OL, Rhee V, Schainker B (1972) Cysteine-induced brain damage in infant and fetal rodents. Brain Res 45:309-313.

Pan Z, Perez-Polo R (1993) Role of nerve growth factor in oxidant homeostasis: glutathione metabolism. J Neurochem 61:1713-1721.

Pileblad E, Magnusson T (1992) Increase in rat brain glutathione follow- ing intracerebroventricular administration of $\gamma$-glutamylcysteine. Biochem Pharmacol 44:895-903.

Pileblad E, Magnuson T, Fornstedt B (1989) Reduction of brain glutathione by L-buthionine sulfoximine potentiates the dopamine-depletion action of 6-hydroxydopamine in rat striatum. J Neurochem 52:978-980.

Pileblad E, Eriksson PS, Hannson E (1991) The presence of glutathione in primary neuronal and astroglial cultures from rat cerebral cortex and brain stem. J Neural Transm 86:43-49.

Puka-Sundvall M, Eriksson P, Nilsson M, Sandberg M, Lehmann A (1995) Neurotoxicity of cysteine: interaction with glutamate. Brain Res 705:65-70.

Raps SP, Lai JCK, Hertz L, Cooper AJL (1989) Glutathione is present in high concentrations in cultured astrocytes but not in cultured neurons. Brain Res 493:398-401.

Rehncrona S, Fobergrova J, Smith DS, Siesjö B (1980) Influence of complete and pronounced incomplete cerebral ischemia and subsequent recirculation on cortical concentrations of oxidized and reduced glutathione in the rat. J Neurochem 34:477-486.

Robinson SR, Schousboe A, Dringen R, Magistretti P, Coles J, Hertz L (1998) Metabolic trafficking between neurons and glia. In: Glial cells: their role in behaviour (Laming PR, Sykova E, Reichenbach A, Hatton GI, Bauer H, eds), pp 83-106. Cambridge: Cambridge UP.

Sagara J, Miura K, Bannai S (1993) Maintenance of neuronal glutathione by glial cells. J Neurochem 61:1672-1676.

Sagara J, Makino N, Bannai S (1996) Glutathione efflux from cultured astrocytes. J Neurochem 66:1876-1881.

Sian J, Dexter DT, Lees AJ, Daniel S, Agid Y, Javoy-Agid F, Jenner P, Marsden CD (1994) Alterations of glutathione levels in Parkinson's disease and other neurodegenerative disorders affecting basal ganglia. Ann Neurol 36:348-355.

Sofic E, Lange KW, Jellinger K, Riederer P (1992) Reduced and oxidized glutathione in the substantia nigra of patients with Parkinson's disease. Neurosci Lett 142:128-130.

Spina MB, Squinto SP, Miller J, Lindsay RM, Hyman C (1992) Brainderived neurotrophic factor protects dopamine neurons against 6-hydroxydopamine and $\mathrm{N}$-methyl-4-phenylpyridinium ion toxicity: involvement of the glutathione system. J Neurochem 59:99-106.

Stole E, Smith TK, Mannings JM, Meister A (1994) Interaction of $\gamma$-glutamyl transpeptidase with acivicin. J Biol Chem 269:21435-21439.

Tate SS, Meister A (1974) Interaction of $\gamma$-glutamyl transpeptidase with amino acids, dipeptides, and derivatives and analogs of glutathione. J Biol Chem 249:7593-7602.

Thompson GA, Meister A (1975) Utilization of L-cystine by the $\gamma$-glutamyl transpeptidase- $\gamma$-glutamyl cyclotransferase pathway. Proc Natl Acad Sci USA 72:1985-1988.

Wüllner U, Löschmann PA, Schulz JB, Schmid A, Dringen R, Eblen F, Turski L, Klockgether T (1996) Glutathione depletion potentiates MPTP and $\mathrm{MPP}^{+}$toxicity in nigral dopaminergic neurons. NeuroReport 7:921-923.

Yudkoff M, Pleasure D, Cregar L, Lin Z-P, Nissim I, Stern J, Nissim I (1990) Glutathione turnover in cultured astrocytes: studies with $\left[{ }^{15} \mathrm{~N}\right]$ glutamate. J Neurochem 55:137-145. 